\title{
An abdominal mass with thrombosis due to inferior vena cava agenesis mimicking lymphoma
}

\author{
Maaike Weijmans $^{1} \cdot$ Rosa Vermeulen $^{1} \cdot$ Fleur S. Kleijwegt $^{1} \cdot$ Henk-Jan Boiten $^{1,2}$ (D)
}

Received: 10 July 2021 / Accepted: 25 July 2021 / Published online: 2 August 2021

(c) The Author(s), under exclusive licence to Springer-Verlag GmbH Germany, part of Springer Nature 2021

\section{Dear Editor,}

A 34-year-old male presented with a 1-week history of low back pain and diffuse abdominal pain without associated symptoms. He had an active lifestyle including bicycle racing. The patient's medical history was unremarkable. He had not been vaccinated with a COVID-19 vaccine. Physical examination revealed no abnormalities except for diffuse abdominal tenderness. Temperature was $38.4{ }^{\circ} \mathrm{C}$ $\left(101.1^{\circ} \mathrm{F}\right)$ with normal remaining vital signs. Laboratory tests showed the values of hemoglobin $11.4 \mathrm{~g} / \mathrm{dL}$, platelets $327 \times 10^{9} / \mathrm{L}$, and leukocytes $8.7 \times 10^{9} / \mathrm{L}$ with normal differentiation. C-reactive protein was $109 \mathrm{mg}$ per liter (reference range $<10$ ). Liver enzymes (including lactate dehydrogenase) and renal function were within normal ranges. A computed tomography (CT) scan revealed an aortocaval soft tissue mass of $8 \times 5 \mathrm{~cm}$ (Fig. 1, panel a, arrows) resembling lymphoma with obliteration of the inferior vena cava. Also, both extensive vascular collaterals and thrombosis were found. Due to the presence of thrombosis, the patient received fraxiparine at a dose of 15.200 IE s.c. daily. Histopathology (measured twice) of the retroperitoneal mass documented atypical chronic fibrosis with neither malignancy nor lymphoma. The treatment was complicated by a psoas muscle hematoma after which the fraxiparine was switched to rivaroxaban $10 \mathrm{mg}$ daily.

Surprisingly, no retroperitoneal mass was found on a CT scan, 4 months later (Fig. 1, panel b). The vascular collaterals remained clearly visible (Fig. 1, panel b, arrows) with an absent inferior vena cava (Fig. 1, panel b, arrow head). All

Henk-Jan Boiten

henkjan_boiten@hotmail.com; h.boiten@erasmusmc.nl

1 Department of Hematology, Erasmus MC, University Medical Center, Rotterdam, The Netherlands

2 Department of Internal Medicine, Erasmus MC, University Medical Center, Doctor Molewaterplein 40, 3015 GD Rotterdam, The Netherlands these findings were consistent with a diagnosis of agenesis of the infrarenal inferior vena cava (IVC).

The etiology by which central vein obstruction occurs can be classified as thrombotic and those that are nonthrombotic. Thrombotic etiologies include inherited thrombophilia, acquired hypercoagulable conditions, immobilization, recent surgery, and testicular tumors. Non-thrombotic obstruction can be categorized into extrinsic (e.g., compression by a tumor, cyst, or aneurysm) and intrinsic causes (e.g., intraluminal device). Although rare, agenesis of the IVC has been reported previously [1-5].

Agenesis of the IVC (IVCA), a type of IVC anomaly, is estimated to occur in $0.0005-1 \%$ of the general population [6]. The embryologic development of the infrarenal IVC is complex deriving from supracardinal veins approximately 6 weeks after conception. It is interesting that our patient presented with thrombosis at the age of 34 years without a history of thrombosis. In a report of 10 patients with IVCA [7], venous thrombosis followed intense physical activity in 8 patients, as was the case in our patient who was active in bicycle racing. We hypothesize that the collaterals (who developed before the venous thrombosis) were unable to assure the increased blood flow due to major physical activity, thereby generating venous stasis and thrombosis.

Due to inadequate drainage of venous blood, IVCA is a risk factor for deep venous thrombosis (DVT). In young ( $<40$ years old) patients with IVCA, the rate of DVT is relatively high (5\%) compared to that of the same patients without IVCA $(0.5 \%)$ [8]. In a study of 97 patients with DVT, a total of 31 patients showed thrombotic occlusion of iliac veins. Five of the 31 patients had an anomaly of the IVC of which only one patient had IVCA [9].

IVCA is a rare but important congenital condition that predisposes to thrombosis. This diagnosis should be considered in the young especially in active male patients presenting with a clinical picture similar to that of the inherited thrombophilias. 
Fig. 1 A Computed tomography (CT) scan shows an abdominal mass (arrows). B CT scan shows extensive vascular collaterals (arrows) and an absent inferior vena cava (arrow head)

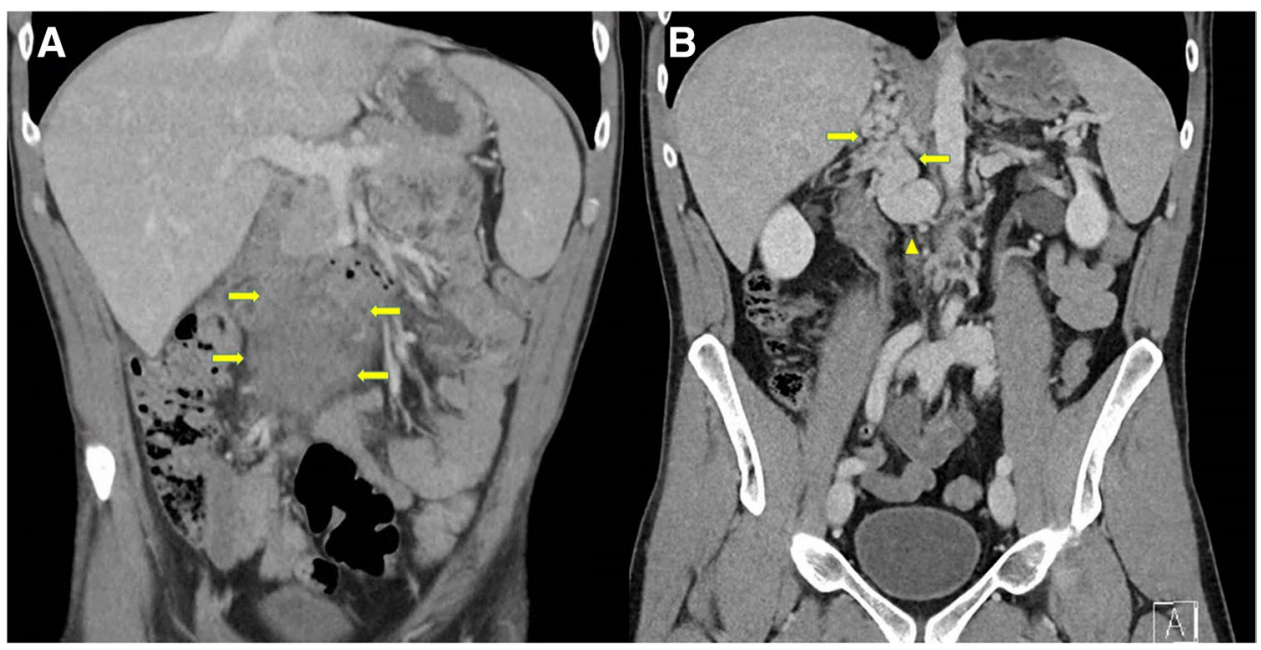

Author contribution MW and HJB performed the research. MW, RV, FSK, and HJB analyzed the data and wrote the paper. HJB supervised the study.

Data availability Data available on request from the authors.

Code availability Not applicable.

\section{Declarations}

Ethical approval All procedures were in accordance with the ethical standards of the institutional and/or national research committee and with the 1964 Helsinki declaration and its later amendments or comparable ethical standards.

Informed consent Informed consent was obtained from the participants included in the study.

Conflict of interest The authors declare no competing interests.

\section{References}

1. Parsa P, Lane JS 3rd, Barleben AR, Owens EL, Bandyk D (2015) Congenital agenesis of inferior vena cava: a rare cause of unprovoked deep venous thrombosis. Ann Vasc Surg 29:1017.e15-18. https://doi.org/10.1016/j.avsg.2015.01.003

2. Thein OS, Braganza Menezes DA, Moran E (2018) Pyrexia of unknown origin: inferior vena cava agenesis. BMJ Case
Rep 23;2018:bcr2018224739. https://doi.org/10.1136/ bcr-2018-224739

3. Man L, Hendricks N, Maitland H (2015) IVC agenesis: a rare cause of deep vein thrombosis. J Thromb Thrombolysis 41:541543. https://doi.org/10.1007/s11239-015-1289-0

4. Nseir W, Mahamid M, Abu-Rahmeh Z, Markel A (2011) Recurrent deep venous thrombosis in a patient with agenesis of inferior vena cava. Int J Gen Med 4:457-459. https://doi.org/10.2147/ IJGM.S21423

5. Pozzi A, El Lakis MA, Chamieh J, Carbonell BB, Villa F (2016) The typical presentation spectrum of deep vein thrombosis associated with inferior vena cava malformations. Thrombosis 2016:4965458. https://doi.org/10.1155/2016/4965458

6. Sneed D, Hamdallah I, Sardi A (2005) Absence of the retrohepatic inferior vena cava: what the surgeon should know. Am Surg 71:502-504

7. Lambert M, Marboeuf P, Midulla M, Trillot N, Beregi JP, Mounier-Vehier C, Hatron PY, Jude B (2010) Inferior vena cava agenesis and deep vein thrombosis: 10 patients and review of the literature. Vasc Med 15:451. https://doi.org/10.1177/1358863X10 391355

8. Ruggeri M, Tosetto A, Castaman G, Rodeghiero F (2001) Congenital absence of the inferior vena cava: a rare risk factor for idiopathic deep-vein thrombosis. Lancet 357:441. https://doi.org/ 10.1016/S0140-6736(00)04010-1

9. Obernosterer A, Aschauer M, Schnedl W, Lipp RW (2002) Anomalies of the inferior vena cava in patients with iliac venous thrombosis. Ann Intern Med 136:37. https://doi.org/10.7326/ 0003-4819-136-1-200201010-00009

Publisher's note Springer Nature remains neutral with regard to jurisdictional claims in published maps and institutional affiliations. 